destruction of the hypothalamus, marked degree of status bullosus and softening of the brain, after 44 hours of artificial respiration. The condition of decreased cerebral blood flow produced clumping of vessels in the brain, but not produce status bullosus, unless irreversible vasopalaysis developed secondarily. On the other hand, these reproducible changes were observed in the cat cerebellar tissue, fixed over 12 hours incubation in normal saline at $38^{\circ} \mathrm{C}$.

These clinical and experimental results suggest that the phenomenon of status ballosus and marked softening of the brain tend to occur easily in the cases of severe vasopalalytic state of the brain with the lesion of the hypothalamus and the upper brain stem, and succeeding prolonged artificial respiration. Furthermore, we discussed histological findings modified by brain edema, impaired cerebral blood flow and/or post-mortem autolysis.

\title{
13. Intracranial Ultrasonic Pulsating Echo and Cerebral Circulation
}

Toshihira Takahashi, Hiroshi Hata, Yasuyoshi Kayama, and Yutaka INABA

Department of Neurosurgery, Tokyo Medical and Dental School

\section{Brain of Immature Animal and Water Intoxication}

\author{
Akira Hirose, Akio Ookuma, \\ Michio Yamaguchi, Takao Taketomo \\ 2nd Department of Surgery, University of Gifu
}

